\title{
@@ OPENACCESS PREVALENCE OF WEAK D PHENOTYPE IN BLOOD DONORS: \\ Check for updates A STUDY FROM TERTIARY CARE HOSPITAL
}

\author{
Mehreen Hameed巛, Fazle Raziq
}

Department of Pathology, Rehman Medical Institute, Peshawar-Pakistan

Address for correspondence: Mehreen Hameed Department of Pathology, Rehman Medical Institute, Peshawar-Pakistan

E-mail: mehreenhameed765@ gmail.com

Date Received: October, $10^{\text {th }} 2020$

Date Revised: July, $20^{\text {th }} 2021$

Date Accepted: July, $24^{\text {th }} 2021$
This article may be cited as Hameed M, Raziq F. Prevalence of weak D phenotype in blood donors: A study from tertiary care hospital. J Postgrad Med Inst 2021;35(2):85-9. https://doi.org/10.54079/ jpmi.35.2.2784.

\section{ABSTRACT}

Objective: To determine the prevalence of weak $D$ phenotype among blood donor population.

Methodology: This cross-sectional review was conducted at Rehman Medical Institute, Peshawar from October 2018 to September 2019 and involved 4361 voluntary blood donors. Blood samples obtained for ABO and Rh D blood grouping were tested through conventional test tube technique. Agglutinating commercially available monoclonal anti-D sera were used for Rh D status detection. Du-testing was performed to identify weak-D phenotype. MS Excel and SPSS version-22 were used hands for statistical analysis.

Results: A total of 4361 blood samples were taken from recruited healthy blood donors (one sample per donor). Out of these 3786 (86.8\%) were Rh D positive while 575 (13.2\%) were Rh D negative. Among Rh D negative cases, three $(0.5 \%)$ were weak-D positive constituting $0.5 \%$ among $\mathrm{Rh} \mathrm{D}$ negatives and $0.06 \%$ from total donors screened.

Conclusion: This study concluded a very small prevalence of weak $\mathrm{D}$ in blood donor population. It is recommended that weak-D phenotype detection in Rh negative donors must be considered as an essential part of blood transfusion investigations to prevent risk of alloimmunization in recipients.

Keywords: Weak D Phenotype; Blood donors; Immunogenic.

\section{INTRODUCTION}

The discovery of $\mathrm{ABO}$ blood group systems was an utmost triumph in the sphere of transfusion medicine which had shed light on the importance and necessity of blood group antigens. ${ }^{1}$ After further exploration, other advancement in the same realm was the recognition of Rhesus system. ${ }^{1,2}$ Although more than 50 Rh antigens have been identified, the most of clinically significant antibodies are caused by the five main antigens - D, C, C, E, and e. .,4 $^{3,4}$ The terms "Rh positive" and "Rh negative" refers to the presence or absence of $D$ antigen. ${ }^{5}$ $\mathrm{Rh} \mathrm{D}$ being the most vital part of Rhesus system, some of its variants like weak-D (also called $D^{4}$ ) and partial D can be very intricate in detecting with routine laboratory procedures. The confirmation of these $D$ variants is vital to ensure the safety of blood transfusion. ${ }^{6} \mathrm{~A}$ proper indirect anti-globulin test (IAT) must be carried out for the detection of weak-D because it cannot be perceived with routine serological methods. The RBCs that test positive after IAT are known as "Weak D". The global prevalence of Rh negativity ranges from 3\% to $25 \%$, whereas the prevalence of weak $D$ antigen is 0.2 percent to $1 \% .{ }^{8}$ Though the number of people who test positive for Weak $D$ is small, but early diagnosis aids in safe blood transfusion. The importance of weak $D$ arise from the fact that transfusion of red cells from a "weak D" donor to a "Rh D negative" recipient might cause allo-immunization and subsequent exposure to such "D positive" red cells can result in a deadly hemolytic transfusion reaction or hemolytic disease of the newborn in a sensitized pregnant female. ${ }^{9,10}$

Thorough understanding of blood group phenotypic distribution is very necessary for blood banks and transfusion services. ${ }^{11}$ For safe blood transfusions, the detection of weak "D" antigen must be an important element of blood grouping and compatibility testing. ${ }^{12}$ Because the weak " $D$ " antigen may not be identified by the immediate spin tube approach, all Rh-negative samples should undergo "Du Testing" for weak "D" antigen detection. ${ }^{11}$ Literature search showed laxity towards weak $D$ detection led to the production of antibodies, causing hemolytic transfusion reactions. ${ }^{9,10}$

By going through various studies, the case in Pakistan is no different regarding detection of weak $\mathrm{D}$. This study determines the prevalence of weak- $D$ in voluntary blood donors in our population as limited data on this topic is available from our region and to the best 


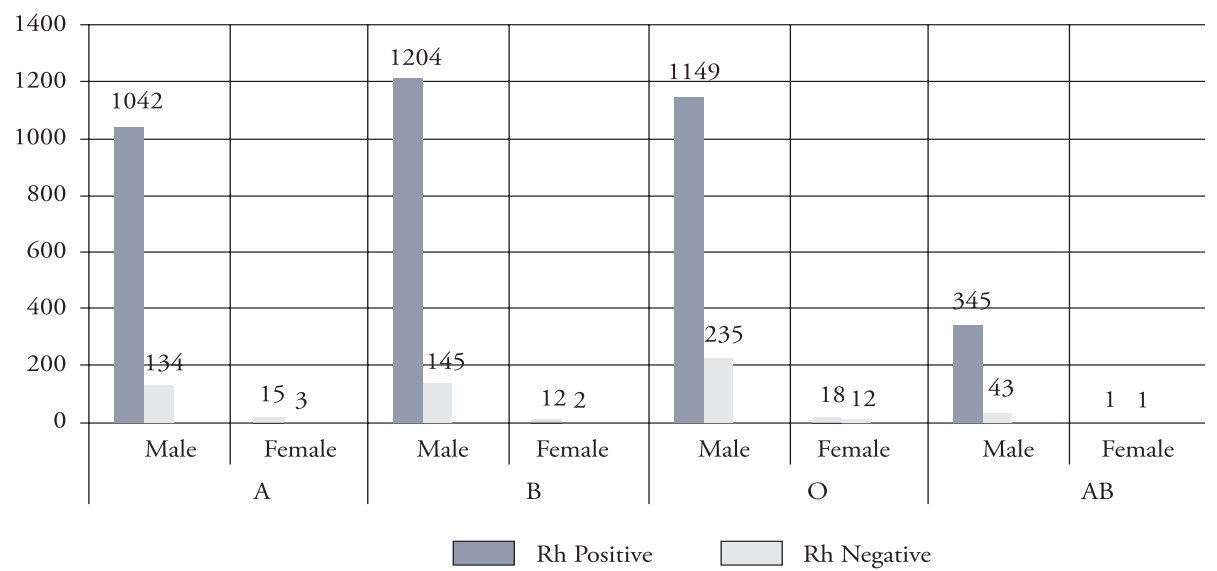

Figure 1: $\mathrm{ABO}$ and $\mathrm{Rh}$ blood groups among blood donors $(\mathrm{n}=4361)$

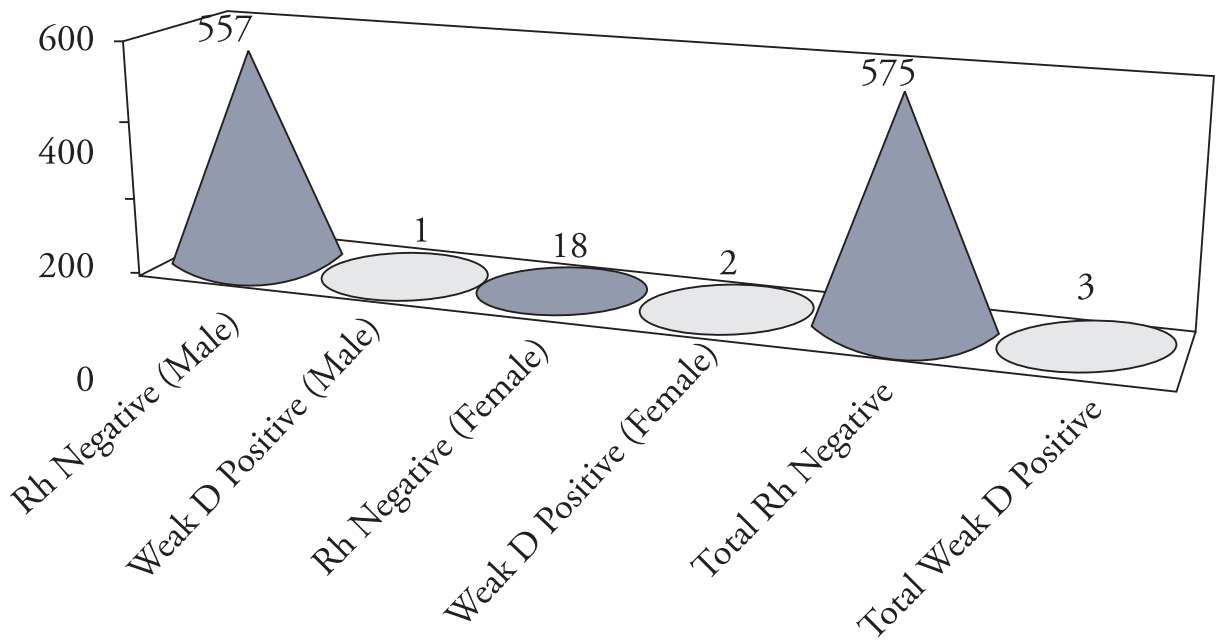

Figure 2: Weak D positive cases in Rh negative blood donors.

of our knowledge this is the first study from Peshawar, Khyber Pakhtunkhwa, Pakistan.

\section{METHODOLOGY}

This cross-sectional research study was carried out at Department of Pathology and Transfusion Services, Rehman Medical Institute, Peshawar, Pakistan from October 2018 to September 2019 after the approval of Ethical committee and management of Blood Bank and Transfusion Services Unit. Total 4361 healthy blood donors were part of this study. By using standard venipuncture technique, two $\mathrm{ml}$ of whole blood samples were withdrawn from each study participant's cubital vein in the lavender top K2- EDTA tube $\left(B D\right.$ vacutainer $\left.{ }^{\circledR}\right)$. All healthy blood donors' samples were subjected for $A B O$ and $R h D$ typing with the help of commercially available monoclonal anti-sera by the test tube method. Samples found to be Rh D negative were further subjected to Du-testing (indirect coomb's technique) for investigation of "weak-D" antigen status. After the addition of equal volume of patient's red cell suspension and anti- $\mathrm{D}$ reagent to a test tube, the process of incubation was carried out for half hour (30 minutes) at $37^{\circ} \mathrm{Celsius.} \mathrm{Following}$ centrifugation the cell button was re-suspended and agglutination was observed macroscopically as well as microscopically. Samples which showed agglutination were labelled Rh-positive while those with the absence of agglutination were rinsed five times with normal saline. Subsequent to last rinse, two drops of anti-human globulin (Coomb's serum) were put in a test tube, mixed and centrifuged at 3400rpm for 15 seconds. Agglutination was observed macroscopically as well as microscopically after centrifugation and any agglutination at this point was documented as "weak-D" positive reaction. Microsoft Excel and Statistical Package for Social Sciences (SPSS) version-22 were used for data entry and interpretation with statistical scrutiny. Frequencies (percentages) were calculated and results were displayed in the form of tables and graphs.

\section{RESULTS}

About 4361 healthy volunteer blood donors were recruited in this study. Male do- 
Table 1: Comparison of prevalence of weak-D in blood donors from different regions of the world including Pakistan.

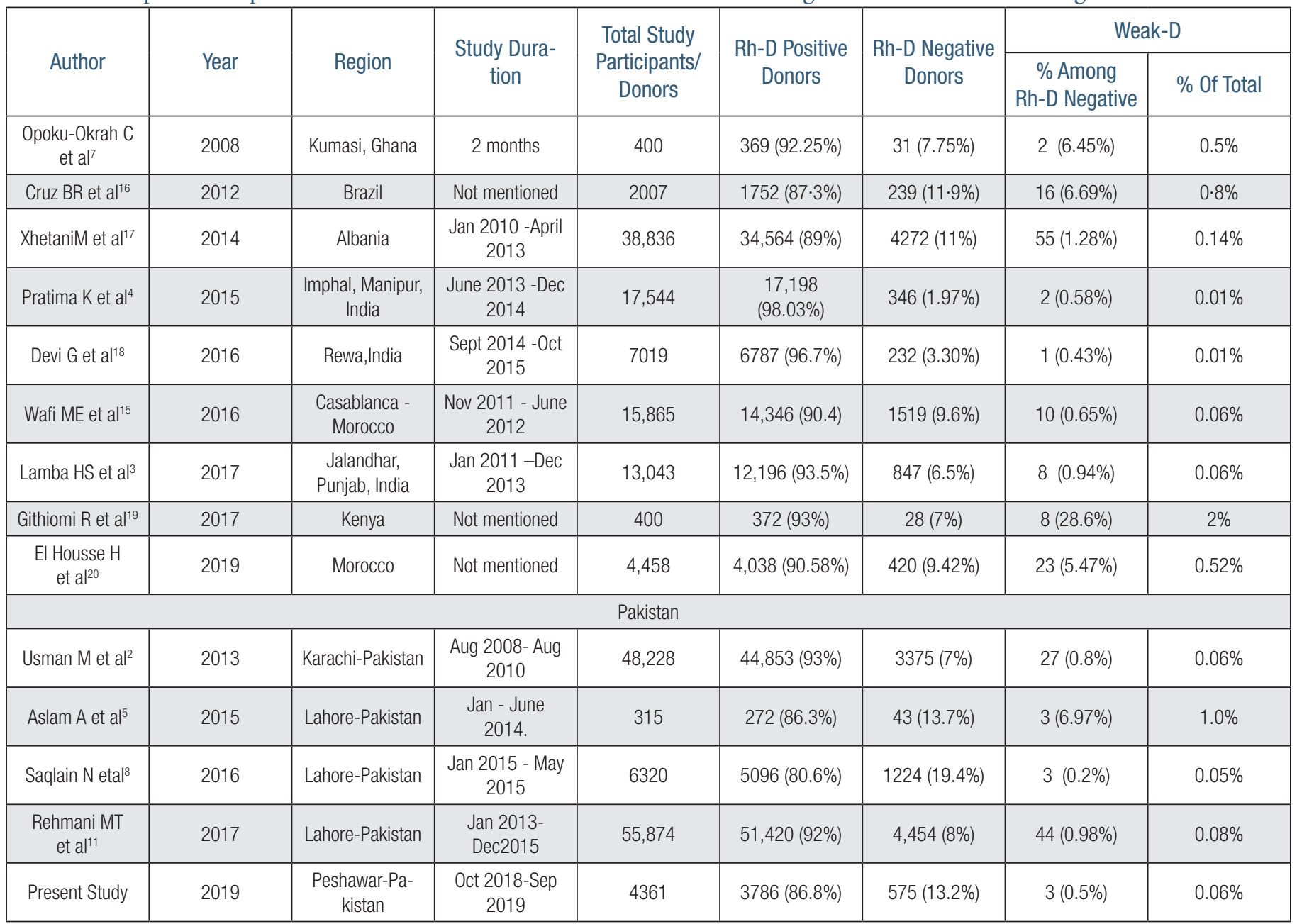

nors dominated with a percentage of $98.5 \%$ $(n=4297)$ while female donors making only $1.47 \%(n=64)$. Distribution of $A B O$ and $R h$ blood groups including weakD are illustrated in figures 1-2.

\section{DISCUSSION}

In the life history of blood transfusions, the unearthing of blood group antigen is one of the profound achievements, in which Karl Landsteiner made a vital breakthrough in the form ABO blood group systemin 1900.2 This discovery of $\mathrm{ABO}$ was a groundbreaking success in organ transplantation and blood transfusion. ${ }^{1}$ In 1939 another cardinal finding by Levine and Stetson in this regards was Rh antigens. ${ }^{6}$ This quantum leap in the form of Rhesus system was a predominant episode in the history of transfusion medicine after ABO. ${ }^{4,6}$ Before the unveiling of Rhesus system, transfusion of blood was a threatening procedure as it was causing transfusion reactions due to anti-D. People having $\mathrm{Rh}$ antigens on their red blood cells are called Rh positive and those who are deficient can be labeled as Rh negative. ${ }^{5}$ Although having a detailed look of its genetic makeup and complexity, more than $50 \mathrm{Rh}$ antigens have been discovered, in which D, C, C, E and e are notably important. The Rhesus (Rh) antigen encodes homologous genes present on chromosome number 1 called RHD and RHCE which are responsible for proteins $D$ and $\mathrm{C}, \mathrm{C}, \mathrm{e}, \mathrm{E}$ respectively. Among these, Rh $D$ being highly immunogenic has major clinical significance in transfusion medicine. ${ }^{13}$

The discovery of Rh $\mathrm{D}$ was very crucial as it helped in ruling out life threatening condi- tions like transfusion reactions and hemolytic disease of newborn (HDN) etc. Thus, as a major antigen of the Rh blood group system, the existence or non-existence of $\mathrm{Rh} D$ on the surface of red blood cells, ascertains its nature of being $R h D$ positive or Rh $D$ negative. ${ }^{6,13}$ Due to its polymorphic nature, almost 200 alleles of RHD gene have been discovered. ${ }^{14} \mathrm{~A}$ term weak $\mathrm{D}$ was deciphered by Stratton in 1946, leading to the explanation of weak $D$ also known as $D^{u}$ which elucidates D-phenotype with weak exhibition of $D$ antigens in quantity as compare to normal Rh D. ${ }^{9,11}$ It's also important to distinguished weak $D$ from partial $D$. Variation in the quantity of $D$ antigen proclaims weak-D while the variation in the quality declares partial $D .{ }^{6}$

Prevalence of weak-D varies worldwide. Being the first study from Khyber Pakh- 
tunkhwa (Peshawar,Pakistan) conducted in one of the major tertiary care center of the province; our results can be considered as symbolic of population of this whole region. In this contemporary study, the prevalence of weak $D$ in our population is under discussion after an extensive investigation of 4361 blood donors. Findings of various previously published studies from different territories of the World including Pakistan and their comparison with the current study are mentioned in Table No. 1. The prevalence of weak-D in present study was found to be $0.06 \%$ of total 4361 blood donors which is similar to the research studies conducted in Casablanca-Morocco by Wafi ME et $\mathrm{al}^{15}$ and in Jalandhar-Indiaby Lamba HS et al ${ }^{3}$. Previous research studies from different regions of the Pakistan showed little variation regarding prevalence of weak-D among blood donors. Frequency of weak-D was found to be $1 \%$, $0.05 \%$ and $0.08 \%$ in studies conducted in Lahore-Pakistan by Aslam A et al ${ }^{5}$, Saqlain $\mathrm{N}$ etal ${ }^{8}$ and Rehmani MT et al1 ${ }^{11}$ respectively. However, a study conducted at Karachi-Pakistan by Usman M et al ${ }^{2}$ in 2013 demonstrates similar results with our study.

In spite of the fact that molecular tests are the decisive answer to limit the disparity of weak-D and D variants, yet in underdeveloped countries, anti-human globulin test is the only ray of hope to unmask weak-D variants, especially for blood donors and women of child bearing age. ${ }^{21}$ The current study is limited by the lack of molecular analysis of weak-D variants. To overcome the limitations of this study, further studies are needed to determine the frequency of weak $D$ in our population by using molecular testing on large sample population which will identify the majority of $D$ variants in our population and also assists immunohematologists in resolving serological discrepancies and developing best anti-D alloimmunization preventive strategies.

\section{CONCLUSION}

This study concluded a very small prevalence of weak $D$ in our region in blood donor population. Although uncommon, misinterpretation of weak $D$ phenotype can become fatal, so it is necessary to check all Rh negative individuals for weak $D$ status as it can precipitate immune response in an individual who lacks Rh-D antigen. Also all health care workers should be well informed of this entity to avoid anti $\mathrm{D}$ alloimmunisation and to ensure safety of blood transfusions.

\section{ACKNOWLEDGEMENTS}

We would like to express our gratitude to blood bank staff of Rehman Medical Institute for their cooperation, assistance during sample collection and technical support in carrying out this study.

\section{口EFERENCES}

1. Basu D, Datta SS, Montemayor C Bhattacharya $P$, Mukherjee $K$, Flegel WA. ABO, Rhesus and Kell antigens, alleles and haplotypes in West Bengal, India. Transfus Med Hemother. 2018; 45(1):62-6.

2. Usman M, Rizwan M, Waheed $U$, Moinuddin SM, Anjum S. Prevalence of weak 'd'antigen in Pakistani population. J Pub Health Bio Sci. 2013; 2(1):169-72.

3. Lamba HS, Kaur K, Kaur K, Vij AS. Prevalence of Weak $D(D u)$ in blood donors in a referral teaching hospital. JDR Clin Trans Res. 2017; 6(2):150912.

4. Pratima K, Barilin P, Avila S, Memtombi DK, Rachandra SK, Nando SK, Barindra SA, Meina SA. Study of Rhesus Status among Blood Donors in RIMS Hospital, Imphal. Int J Health Sci Res. 2015; 5(7):111-4.

5. Aslam A, Azmi R, Sheikh MZ, Javaid I. Frequency of weak expression of ' $D$
ALLELE' among healthy blood donors. Pak J Physiol. 2015; 11(3):22-4.

6. Kabiri Z, Benajiba M, Hajjout K, Dakka N, Bellaoui H. Testing for partial $\mathrm{RhD}$ with a D-Screen diagast kit in moroccan blood donors with weak D expression. J Blood Transfus 2014; 14:1-4. https://doi. org/10.1155/2014/204301.

7. Opoku-Okrah C, Amidu N, AmoahSakyi S. Detection of Weak D (Du) Phenotype among Rh-D negative males and females in Kumasi, Ghana. J Sci Technol 2008; 28(3):34-40.

8. Saqlain N, Ahmed A, Fateen T, Ahmed N. D antigen; chances of finding weak $\mathrm{d}$ antigen and re-evaluation of its clinical significance as a routine blood bank procedure. Professional Med J. 2016; 23(11):1395-9.

9. Kumar H, Mishra DK, Sarkar RS, Jaiprakash M. Difficulties in immunohaematology: The weak D antigen. Med J Armed Forces India. 2005; 61(4):348-50.

10. Noizat-Pirenne $F$, Verdier $M$, Lejealle A, Mercadier A, Bonin P, et al. Weak D phenotypes and transfusion safety: where do we stand in daily practice? Transfusion. 2007; 47(9):1616-20.

11. Rahmani MT, Ahmad M, Saeed M, Hussain S, Hanif A, Rashid N. Determination of Weak "D" Antigen among Rhesus Negative Pakistani Blood Donors. Ann Pak Inst Med Sci. 2016; 12(3):131-35.

12. Schmidt LC, Castilho L, Vieira OV, Sippert E, Gaspardi AC, Martins ML, et al. Impact of a confirmatory RhD test on the correct serologic typing of blood donors. Rev Bras Hematol Hemoter. 2015; 37(5):302-5.

13. Makroo R, Gupta R, Bhatia A, Rosamma NL. Rh phenotype, allele and haplotype frequencies among 51,857 blood donors in North India. Blood Transfus. 2014; 12(1):36.

14. Flegel WA. Molecular genetics and 
clinical applications for RH. Transfus Apher Sci. 2011; 44(1):81-91.

15. Wafi ME, Housse HE, Nourichafi N, Bouisk K, Benajiba M, Habti N. Prevalence of weak $D$ phenotype among $D$ negative $\mathrm{C} / \mathrm{E}+$ blood donors in Morocco. Int J Blood Transfu Immunohematology. 2016; 6:3-7.

16. Cruz BR, Chiba AK, Moritz E, Bordin J0. RHD alleles in Brazilian blood donors with weak $D$ or D-negative phenotypes. Transfus Med. 2012; 22(2):84-9.

17. Xhetani M, Seferi I, Férec C, Zoraqi G,
Fichou Y. Distribution of Rhesus blood group antigens and weak $D$ alleles in the population of Albania. Blood Transfus. 2014; 12(4):565.

18. Devi G, Singh UR, Garg A. Detection of weak RhD (Du) phenotype among blood donors. Ind J Basic Appl Med Res. 2016; 5(4):135-8.

19. Githiomi R, Waiganjo N, Muna K. The heterogeneity and distribution patterns of $A B O$ and $R H D$ phenotypes in the voluntary blood donors of Kenya. J Clin Immunol Immunopathol Res. 2017; 8(1):1-7.
20. El Housse H, El Wafi M, Ouabdelmoumene Z, Zarati F, Alid R, Nourichafi N, Bouisk K, et al. Comprehensive phenotypic and molecular investigation of RhD and RhCE variants in Moroccan blood donors. Blood Transfus. 2019; 17(2):151.

21. Flegel WA, Denomme GA, Yazer $M H$. On the complexity of $D$ antigen typing: a handy decision tree in the age of molecular blood group diagnostics. J Obstet Gynaecol Can. 2007; 29(9):746-52.

\section{Author's Contribution}

MH conceptualized and designed the study, acquisition of data with analysis and interpretation of data, drafting of the article and final approval of the manuscript. FR critically revised, proof read and supervised the study. Authors agree to be accountable for all aspects of the work in ensuring : that questions related to the accuracy or integrity of any part of the work are appropriately investigated and resolved.
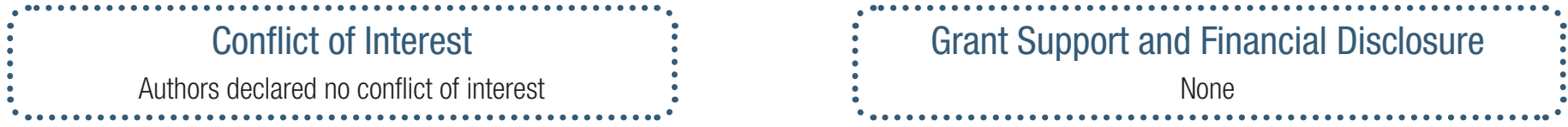

\section{Data Sharing Statement}

The data that support the findings of this study are available from the corresponding author upon reasonable request. 